\title{
Establishment Of Highly Precise CAE Analysis Model Using Automotive Bolts
}

Takashige Takahashi, Aoyama Gakuin University, Japan Toshiya Ueno, Aoyama Gakuin University, Japan Manabu Yamaji, Aoyama Gakuin University, Japan Kakuro Amasaka, Aoyama Gakuin University, Japan

\begin{abstract}
In this paper, the authors propose a model for a highly precise CAE analysis approach, which is intended to contribute to the regeneration of development and design. This model has been applied with significant results in making proposals for bolt tightening behavior analysis, which continues to be an area of concern.
\end{abstract}

Keywords: development design, Highly Precise CAE Analysis Model, CAE analysis of bolt tightening behavior

\section{INTRODUCTION}

Capan's automotive industry is currently aiming to achieve worldwide uniform quality and production at optimum locations in order to ensure survival in today's harsh economic climate. Amidst the rapidly changing management technology situation and worldwide quality competition, it is now imperative for automobile manufacturers to take the initiative in offering highly reliable leading-edge products to enhance customer value if they are to prevail in the global market.

Specifically, it is considered necessary to change over from conventional repetitive evaluation-based development involving a prototyping and testing process (confirmation of actual products for improvement) to predictive evaluation-based methods employing CAE (Computer-Aided Engineering), which is capable of accurately producing equivalent evaluations through statistical simulation (Amasaka,2007a, 2008: Yamaji and Amasaka, 2008: Ueno, et al., 2009).

Thus, in this paper, the authors propose a model for a highly precise CAE analysis approach, which is intended to contribute to the regeneration of development and design. In order to demonstrate its effectiveness, the model has been applied with significant results in making proposals for bolt tightening behavior analysis, which continues to be an area of concern.

\section{RESEARCH}

\subsection{Proposal of "Automotive Intelligence CAE Methods"}

In order to create intelligent $\mathrm{CAE}$ software for realizing quality assurance independent from prototyping and experimental evaluation, the following must be fully clarified, as shown in Figure 1(Amasaka, 2007a): (1) Has the problem been properly confirmed with the actual product? (2) Has the theory needed for solving the problem been established? (3) What type of numerical simulation model should be applied? (4) What type of calculation method should be adopted? (5) What type of calculator needs to be selected? For realization of highly reliable CAE analysis, solutions to problems, such as the following, must be found: (i) how to match the results of simulation and data of the actual vehicle, (ii) how to conduct DR without the actual machine on hand, and (iii) how to prevent errors and omissions in evaluation. Without overcoming these problems, reform in the development design process from "development through real object confirmation and improvement" to "prediction evaluation oriented development" will not progress (Amasaka 2008). 


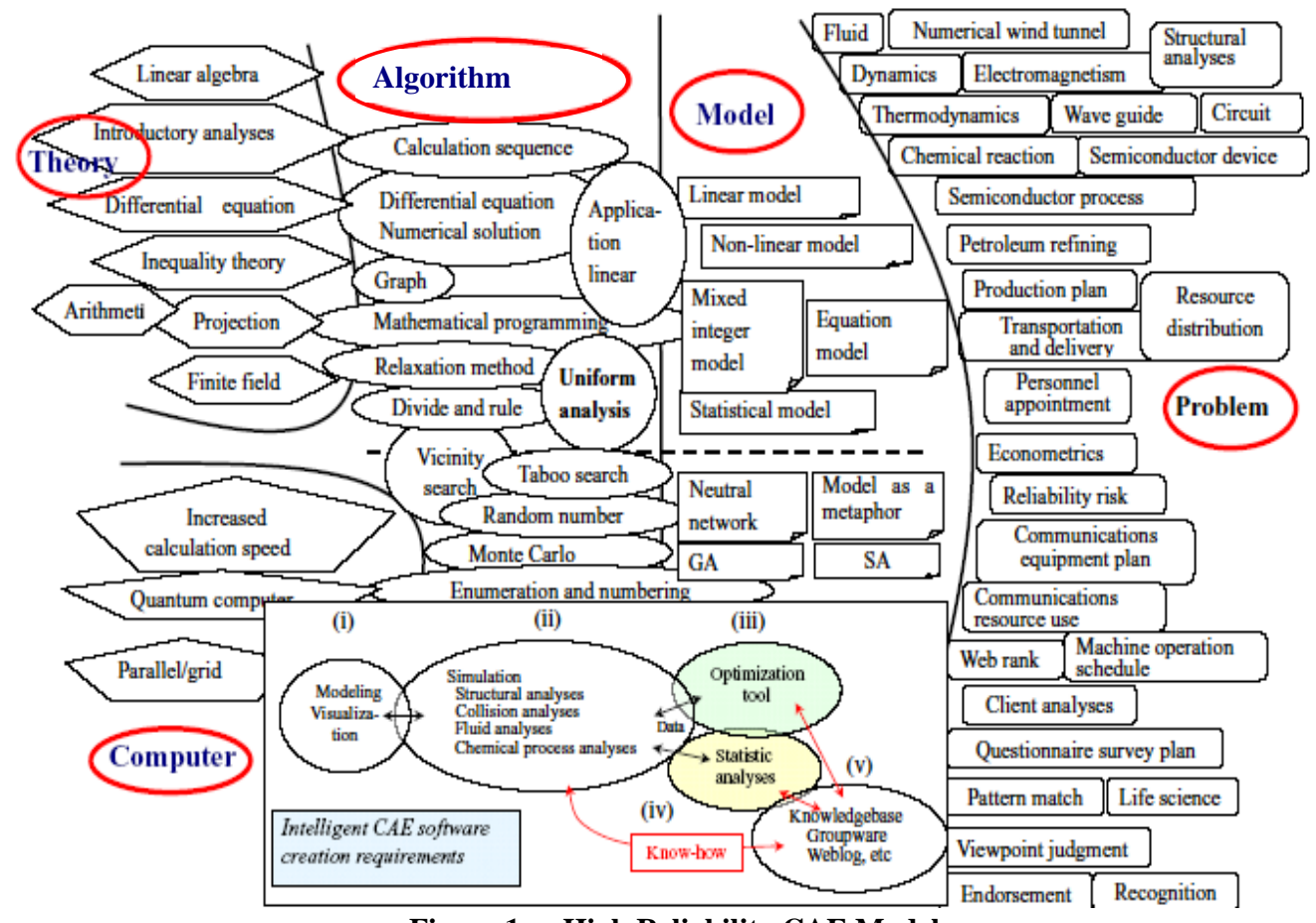

Figure 1: High Reliability CAE Model

\subsection{Proposal of "Total Intelligence CAE Management Model"}

Following the above, the author proposes for establishment of "Automotive Intelligence CAE Methods" (Amasaka 2007b) shown in Figure 2, which contributes to high quality assurance as well as QCD simultaneous achievement in automobile development design. As shown in this figure, many manufacturers are aware of the gap between evaluations of actual vehicles and CAE; and not fully confident in CAE results, they prefer to conduct Step (I) survey tests with actual vehicles rather than CAE evaluation. Even among leading corporations, Step (II) CAE utilization is limited to relative evaluation.

The authors noticed a situation where, as shown in the figure, the application ratio of CAE to actual vehicles is $25 \%$ for surveys and about $50 \%$ for relative evaluation, revealing the dilemma that the effectiveness of CAE invested for reduction in development time has not been fully utilized. Based on the above, in Step (III), as seen in the figure, the mechanism of the pending technical problem was clarified through visualization technology, and the technical knowledge, which enables absolute evaluation through the creation of generalized models, was incorporated in the CAE software.

As a result, it was confirmed that the accuracy of CAE analysis had improved and the application ratio of CAE had increased to $75 \%$. Based on the technical analysis derived from Steps (I) to (III), Step (IV) further incorporated a robust design, which takes into consideration the influential factors and contributing ratio needed for optimal design, thus enhancing the accuracy of CAE calculation and demonstrating a remarkable increase in the ratio of CAE application. 


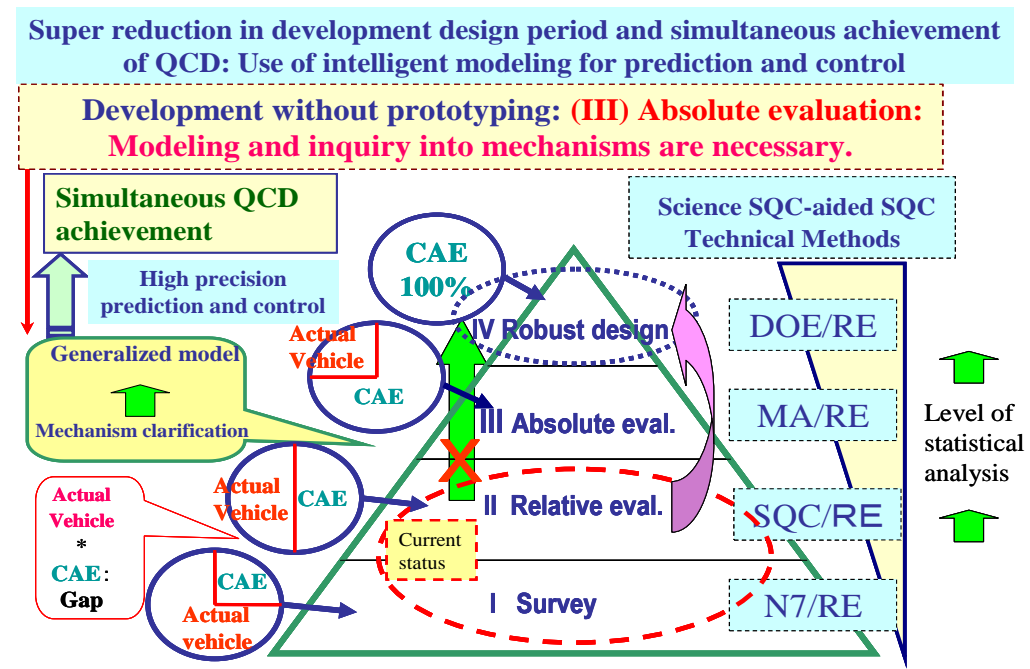

Figure 2: Total Intelligence CAE Management Model

Against the above background, as a fundamental solution to the automotive development and designing problem, the authors created "Intelligence CAE Management System Approach" (Amasaka 2007b), as indicated in Figure 3. As seen in the figure, first, the (A) actual vehicle and experiment (meaning bench evaluation tests using actual vehicles and parts) visualizes the dynamic behavior (tricky mechanism) of the problems. Next, by means of the (B) factorial analysis in which the unique technology and empirical technology are combined together with N7, $\mathrm{RE}, \mathrm{MA}$, and DE, the latent factors, which induce oil leakage, are investigated using actual vehicles and experiment procedures in an effort to clarify the mechanism (Yamaji and Amasaka 2008).

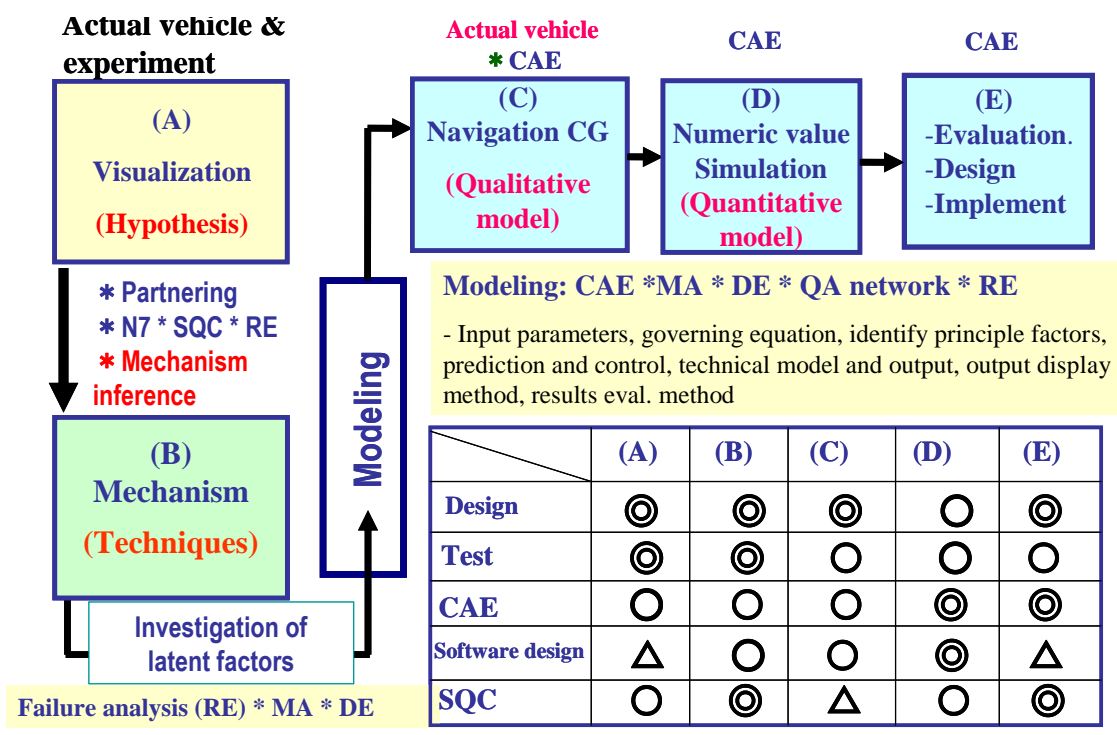

Figure 3: Intelligence CAE Management System Approach

Based on the knowledge obtained through the above steps, as well as the navigation process using CG (Computer Graphics) created by a combination of (C) experiments and CAE, qualitative modeling of problems was conducted. Furthermore, for the purpose of accurately reproducing the mechanism, which has been grasped by an inductive approach of visualization experiments, quantitative modeling is conducted by means of (D) numeric value 
simulation. In the final stage, the (E) differential (gap) between the evaluation results of actual vehicles and experiment procedures (absolute value) and those of the CAE analysis (simulation value) was confirmed.

The target of the differential ratio of analysis precision is between $1 \%$ and $2 \%$. To achieve such a target, an all-out collective partnering is indispensable among the chief engineers ( $\bigcirc)$, collaborating engineers $(O)$, and assistants $(\triangle)$ who are involved in the design, testing, CAE analysis, CAE software development, and SQC throughout the process stages from (A) to (E) indicated in the figure.

Thus, this study uses visualization technology at the absolute evaluation stage to clarify the mechanisms involved in current technical problems and proposes a model for a highly reliable CAE analysis approach to enable absolute evaluation through the creation of generalized models.

\section{PROPOSAL OF A HIGHLY RELIABLE CAE ANALYSIS MODEL}

In order to minimize discrepancies in results obtained from testing of actual products and CAE, it is necessary to properly formalize the expertise of many technical analysts required for CAE analysis. A proposed model demonstrating the approach necessary for highly precise CAE analysis is shown in Figure 4.

The following four steps are proposed in order to achieve a high level of reliability in development and design: (1) surveys, (2) relative evaluation, (3) absolute evaluation, and (4) robust design. Issues remain concerning (3) absolute evaluation if CAE analysis is to guarantee high quality development and design and help enable the simultaneous achievement of QCD (Quality, Cost, and Delivery). Therefore, the authors propose the use of the highly reliable CAE analysis approach to enable highly precise absolute analysis.

Functional failures are a recurring cause of market claims in automotive development and design, making it necessary to clarify relevant technical issues such as the reasons and mechanisms by which such failures occur. This should be done according to the following steps.

Step 1 - Extracting the Problem: Investigate and analyze the causal relationships that become apparent and infer the mechanisms through which failures occur, employing the latest SQC (Statistical Quality Control) methods backed up by expertise accumulated through the cooperative efforts of internal and external specialists.

Step 2 - Visualizing the Problem: Test actual products demonstrating the failure mechanisms to visualize the dynamic behavior of the problem. In order to accurately analyze the failure and its causes, it is necessary to uncover any underlying factors that were not evident from previous findings and may have been overlooked. A logical reasoning process must be applied to demonstrate the mechanisms of the failure, employing tools and principles such as the seven New SQ tools (N7), Statistical Quality Control (SQC), Reliable Engineering (RE), Multivariate Analysis (MA), and Design of Experiments (DE).

Step 3 - Testing \& CAE Analysis: Consolidate the findings and apply statistical simulation to create a two-dimensional model integrated on a qualitative level where the visualization produced through actual testing can be reproduced. When creating this two-dimensional model, it is necessary to conduct tests to produce a model (qualitative modeling) of the causal relationships involved in undefined failure mechanisms. Precise calculation methods, analysis models and algorithms must be properly selected in order to clarify boundary conditions and contact situations and enable highly accurate statistical simulation. It is essential to use such tools to minimize discrepancies between actual testing and CAE absolute evaluation. The findings from such analysis should then be used for more detailed three-dimensional analysis.

Step 4 - Highly Reliable CAE: Conduct accurate testing of actual products based on the findings from step 3 to gain a more explicit understanding of the failure mechanisms. Consolidate the findings from the processes involved and conduct statistical simulation (quantitative modeling) with a high level of credibility to enable the prediction and control of absolute values. 
Step 5 - Predictive Evaluation CAE Analysis: In this final step, identify the primary factors from the results of the statistical simulation in step 4 to be used for prediction and evaluation. The new findings obtained, design concepts, and formulation models should be shared and disseminated. Utilizing models with a higher level of analytic accuracy enables an advance from relative evaluation to absolute evaluation of analysis results in the actual development process. The authors believe that this will lead to the future establishment of design frameworks involving predictive evaluation.

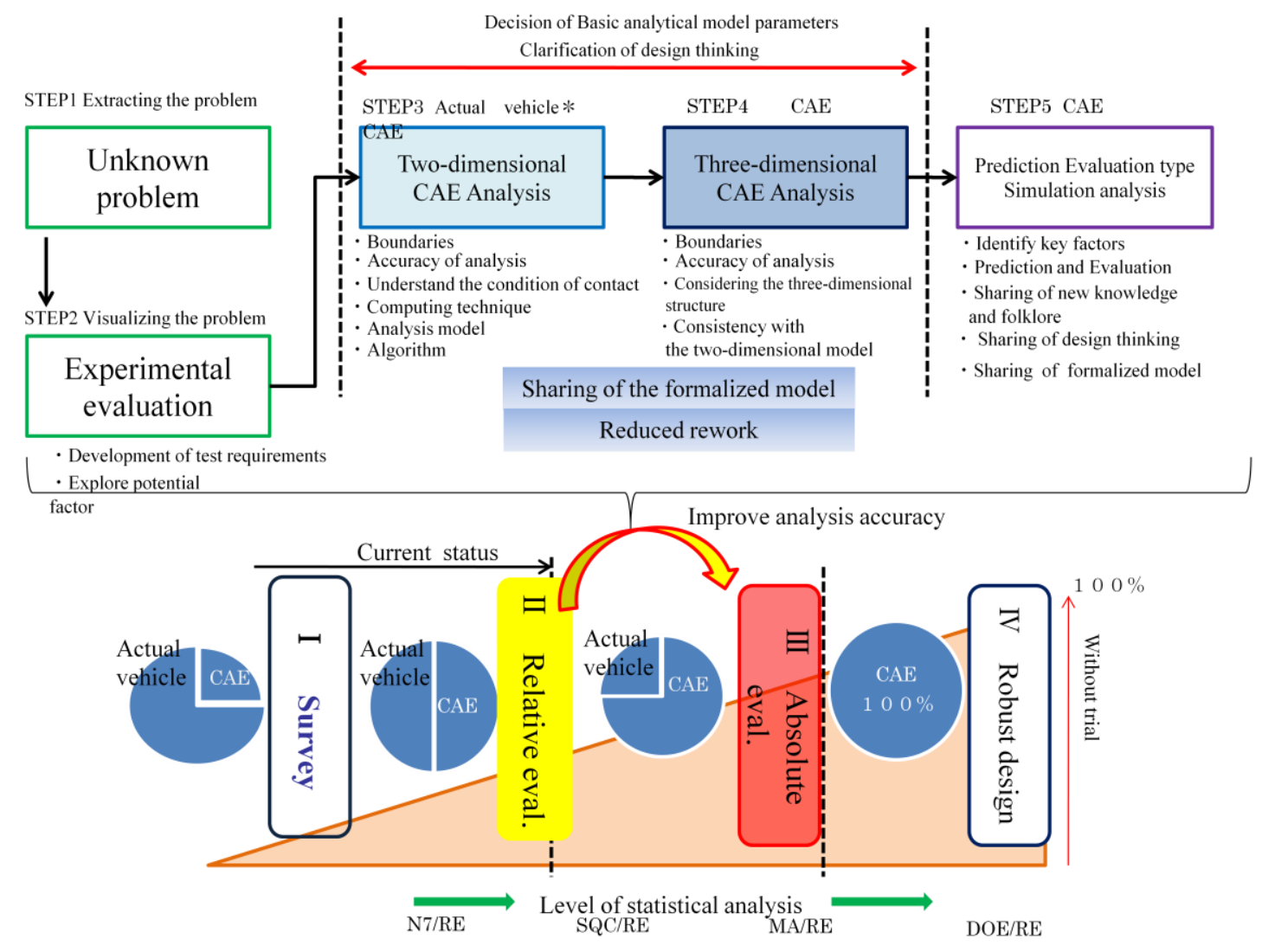

Figure 4: Highly Precise CAE Analysis Model

\section{BOLT TIGHTENING EXPERIMENT AND CAE ANALYSIS OF BOLT TIGHTENING BEHAVIOR}

This section illustrates a case where the highly precise CAE analysis model was used in the CAE analysis of bolt tightening behavior.

\subsection{Step1 - Extracting the Problem and the Technological Elemental Analysis of the Bolt Tightening}

Figure5 shows the following. Relevant issues for conducting simulation of physical chemical phenomena (i) are: (1) the relationship between axial force and torque, (2) the dynamic friction coefficient and static friction coefficient, and (3) contact surface pressure on the threaded portion and bearing surface. Models for solving these issues (ii) are: (1) the dynamic elemental model, (2) the elastic-plastic model, (3) the contact model, and (4) the material component rule model, Useful algorithms (iii) are: (1) the finite element method and (2) the dynamic analysis method. (iv) Suitable theoretical equations are: (1) structural mechanics, and (2) equation of equilibrium. Finally (V) examples of creative solutions for ensuring precision and realistic time calculation are: (1) the temporal 
integration method, (2) the matrix function, (3) the penalty method, and (4) the Lagrange multiplier method. These are to be utilized to compose a highly reliable CAE analysis model (Baggerly, 1996, Gamboa and Atrens, 2003, Leea, et al., 2005).

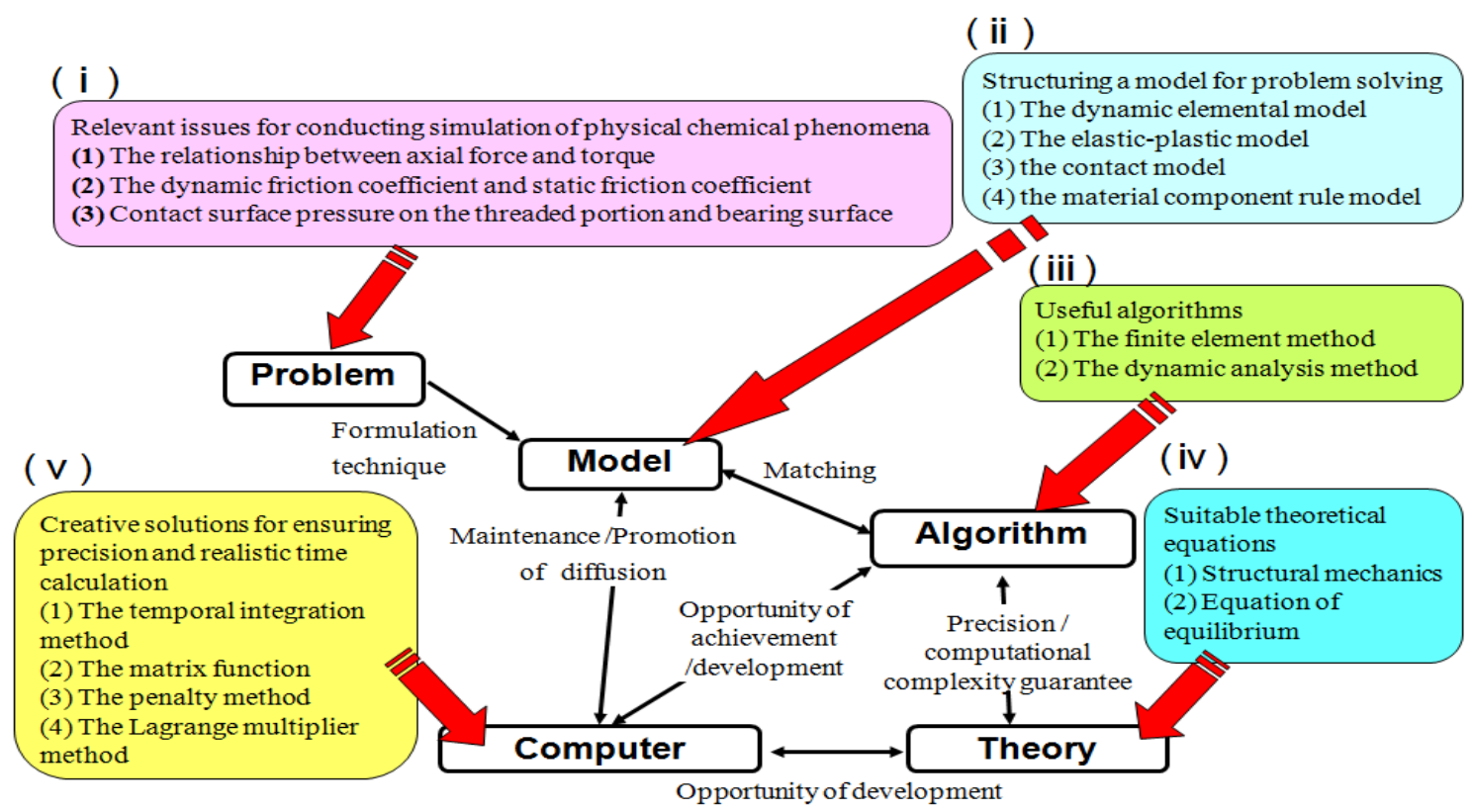

Figure 5: The Technological Elemental Analysis of the Bolt Tightening

\subsection{Step2 - Visualizing the Problem and Bolt Tightening Experiment}

A strain gauge is attached to the material to which hexagon flange bolts are tightened, and an experiment was conducted as shown in Figure 6 in order to confirm an important parameter of the friction coefficient based on the dynamic behavior of "angle * torque * axial force" of (1) the threaded portion and (2) bearing surface (Izumi, et al., 2004,Aragóna, et al., 2005).
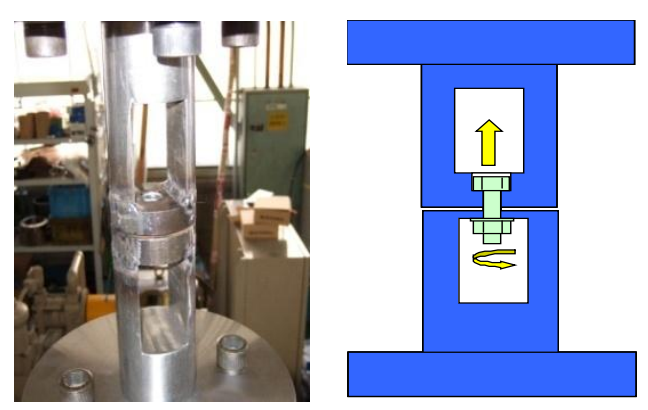

(1) Measuring the friction coefficient of the threaded portion
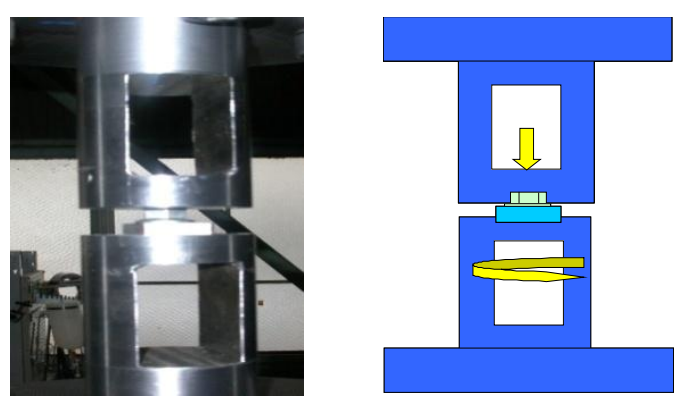

(2) Measuring the friction coefficient of the bearing surface

Figure 6: Measuring the Friction Coefficient to Tighten Bolts

The bolt tightening experiment was conducted using "hexagon flange bolts and hexagon nuts with flange," which are actually used in the automotive industry for commercial use. The friction coefficient of the threaded portion (1) was measured using a 2-axial fatigue testing machine, which is capable of applying axial force and twist force at the same time. 
The bolt head was pulled with the force of $20 \mathrm{KN}, 40 \mathrm{KN}$ and $60 \mathrm{KN}$, conducting the experiment for each tensile force five times in total, while torque and axial force were measured. The friction measurement for coefficient of the bearing surface (2) was similarly conducted using the 2-axial fatigue testing machine, applying a compression load of $20 \mathrm{KN}, 40 \mathrm{KN}$ and $60 \mathrm{KN}$ onto the substrate while rotating the nut so as to confirm the torque and axial force.

Based on the results of the friction coefficient measurement of (1) the threaded portion and (2) the bearing surface, torque and axial force were obtained. Applying theoretical equations to these experiment results, the friction coefficients were calculated.

As an example, the friction coefficients of the threaded portion and bearing surface, when the tensile force and compression load in the axial direction are both $60 \mathrm{KN}$ at the angle of $40^{\circ}$, are shown in Table 1 . Using these calculated friction coefficients, bolt tightening simulation was conducted and the relationship between simulative axial force and torque was confirmed (Sakai, 2000, Suzuki, 2005, Tanaka, et al., 1981).

Table 1: The Friction Coefficients

\begin{tabular}{|c|c|c|c|c|c|}
\hline \multicolumn{3}{|c|}{ The Friction Coefficient of the Threaded Portion } & \multicolumn{3}{|c|}{ The Friction Coefficient of the Bearing Surface } \\
\hline $\begin{array}{c}\text { Angle } \\
\text { (deg) }\end{array}$ & $\begin{array}{c}\text { Torque } \\
\text { (Nm) }\end{array}$ & The friction coefficient & $\begin{array}{c}\text { Angle } \\
\text { (deg) }\end{array}$ & Torque (Nm) & The friction coefficient \\
\hline 40 & 18.63 & 0.02 & 40 & 215.26 & 0.35 \\
\hline 40 & 23.05 & 0.03 & 40 & 221.63 & 0.37 \\
\hline 40 & 35.30 & 0.06 & 40 & 198.09 & 0.33 \\
\hline 40 & 28.44 & 0.04 & 40 & 208.39 & 0.34 \\
\hline 40 & 32.85 & 0.05 & 40 & 226.53 & 0.37 \\
\hline
\end{tabular}

\subsection{Step 3 - Statistical Simulation Using A 2d Finite Element Model}

This analysis utilizes the axis symmetry two-dimensional model. The analysis parameters are the same as the experiment parameters. Regarding the analysis parameters, axial force is applied to part of the upper substrate and the bolt bounded from all directions in the same way as it is applied to part of the bolt head and lower substrate. The friction coefficient was calculated by conducting experiments on parts of the contact bearing surfaces and the contact surface of the threaded portions, and then inputted into CAE simulation software. In addition, the authors compared the relationship between torque and axial force using the results from the experiment and simulation analysis.

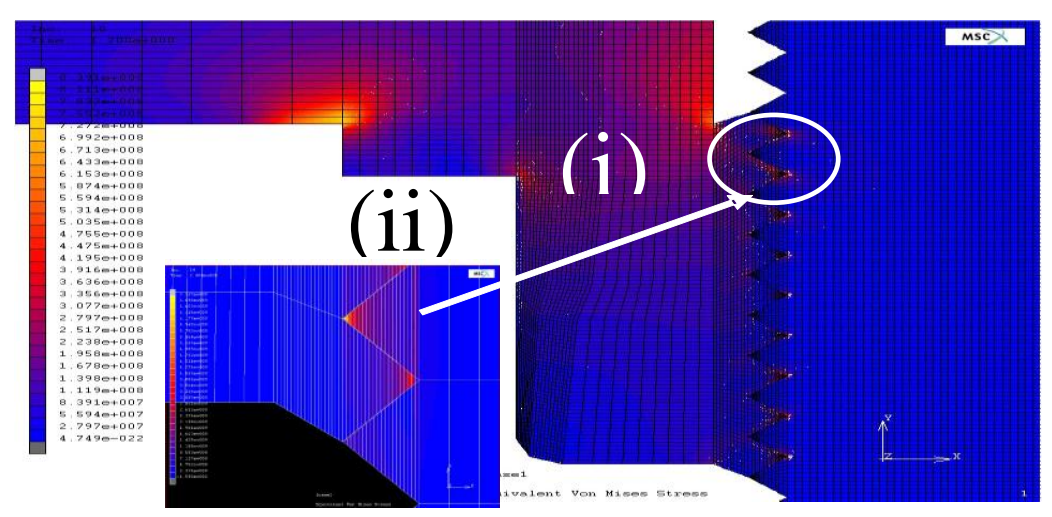

Figure7: Analysis Result of 2D Finite Element Model

As can be seen in the Figure7, (i) the high stress exists at the nut and the base metal boundary. In addition, (ii) the high stress exists part of the helical structure. 


\subsection{Step 4 - Statistical Simulation Using A 3d Finite Element Model}

Figures 8 and 9 show 3D finite element models simulating the helical structure of the threaded portion. In the diagrams, the area around the bolt and nut and the contact sections are represented by a fine mesh compared to the 2D finite element model (figure 7). The analysis process involves the following steps: (1) Place parts to be clamped ( 2 substrates) between the bolt and nut, (2) Apply axial force to the bolt and determine the distribution of stress during contact, (3) Fix the edge of the lower substrate and apply upward perpendicular force (axial force) to the upper substrate, and (4) Determine the pressure on the contact surface (bolt/nut bearing surfaces and between the bolt crest and nut).

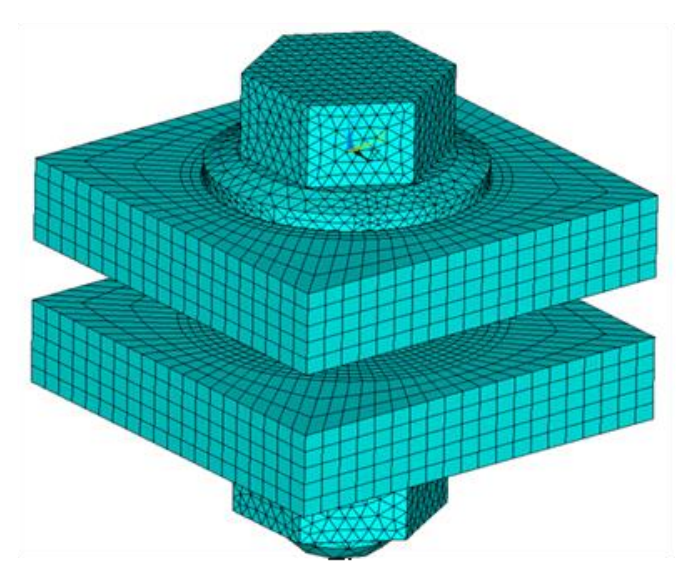

Figure 8: 3D Finite Element Model
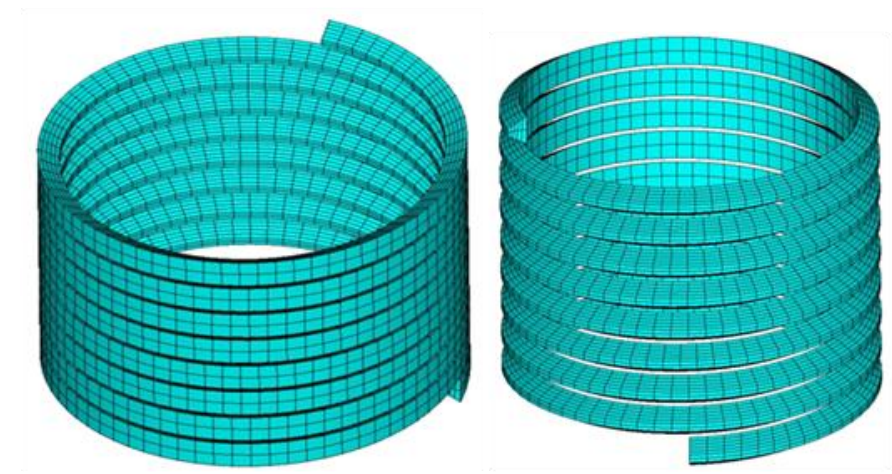

Figure 9: 3D Finite Element Model of the Helical Structure

\subsubsection{Analysis of the Area around Bolt Flanges}

The results of analysis, when axial force was applied to the upper substrate in a perpendicular direction, are shown in Figures 10 and 11. The diagrams show that there is a greater degree of stress on the surface of the substrate in contact with the bolt flange than the periphery of the substrate. This stress distribution shows that stress is concentrated in the perpendicular section between the bolt head and the flange, making it possible to examine more thoroughly the results of analysis conducted using the 2D axial symmetry model in 4.3 (figure 7).

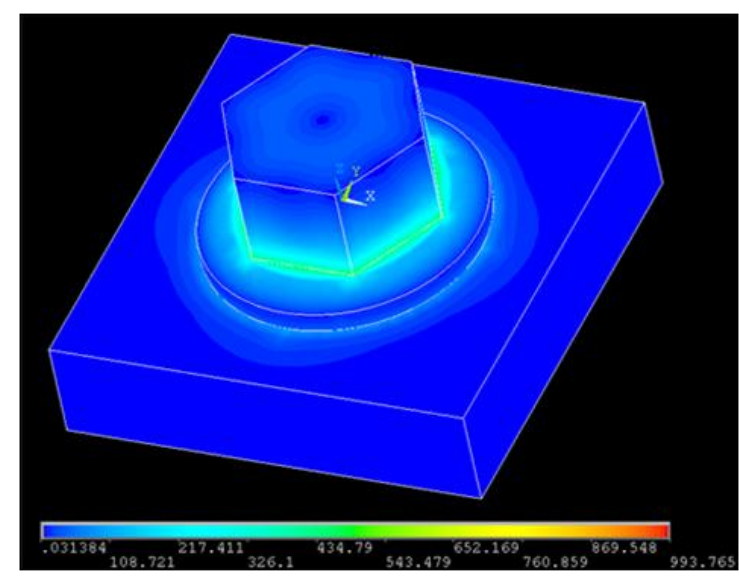

Figure 10: Stress of the Surface of the Substrate

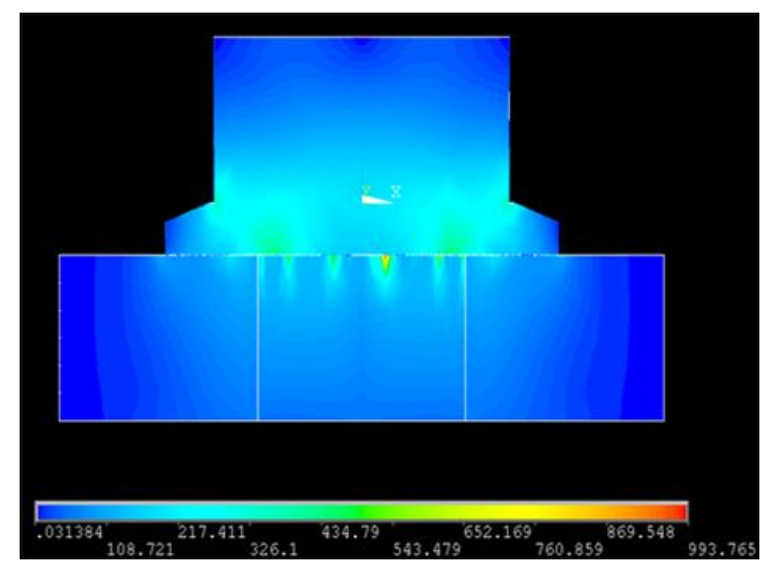

Figure 11: Stress of the Surface of the Substrate (Cross-section) 


\subsubsection{Analysis of the Area around Thread Contact Points}

Figure 12 shows a stress diagram (cross-section) with the thread contact section enlarged. It can be seen from the diagram that the lower section has a higher stress value than the upper section.

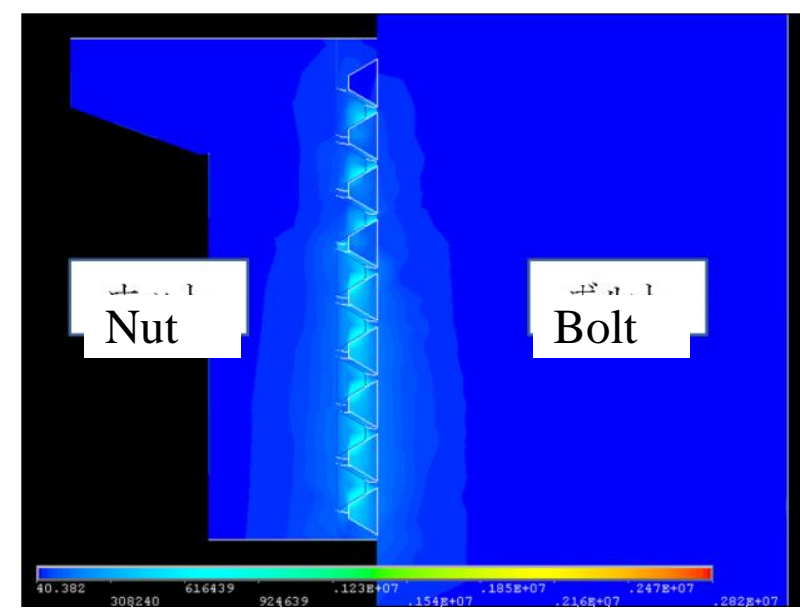

Figure 12: Stress of the Thread Contact Section

\section{$\underline{\text { Comparison of Two-dimensional and Three-dimensional CAE Analysis }}$}

The results of three-dimensional CAE analysis show inconsistent crest stress values (higher stress values at the top of the threaded section than the bottom) in contrast to the stress distribution (mostly consistent stress values for each thread crest) obtained from a 2D axial symmetry model.

The helical structure and trapezoidal shape of the threaded section is likely to be a contributing factor. Analysis using 3D models takes into account the helical structure and models are constructed so that the trapezoidal shape of the threaded section conforms to JIS standards. Such models show that stress probably occurs in the lower part of the nut's threaded section because a perpendicular axial load is applied to the upper substrate and force therefore acts upon the bolt in the same direction.

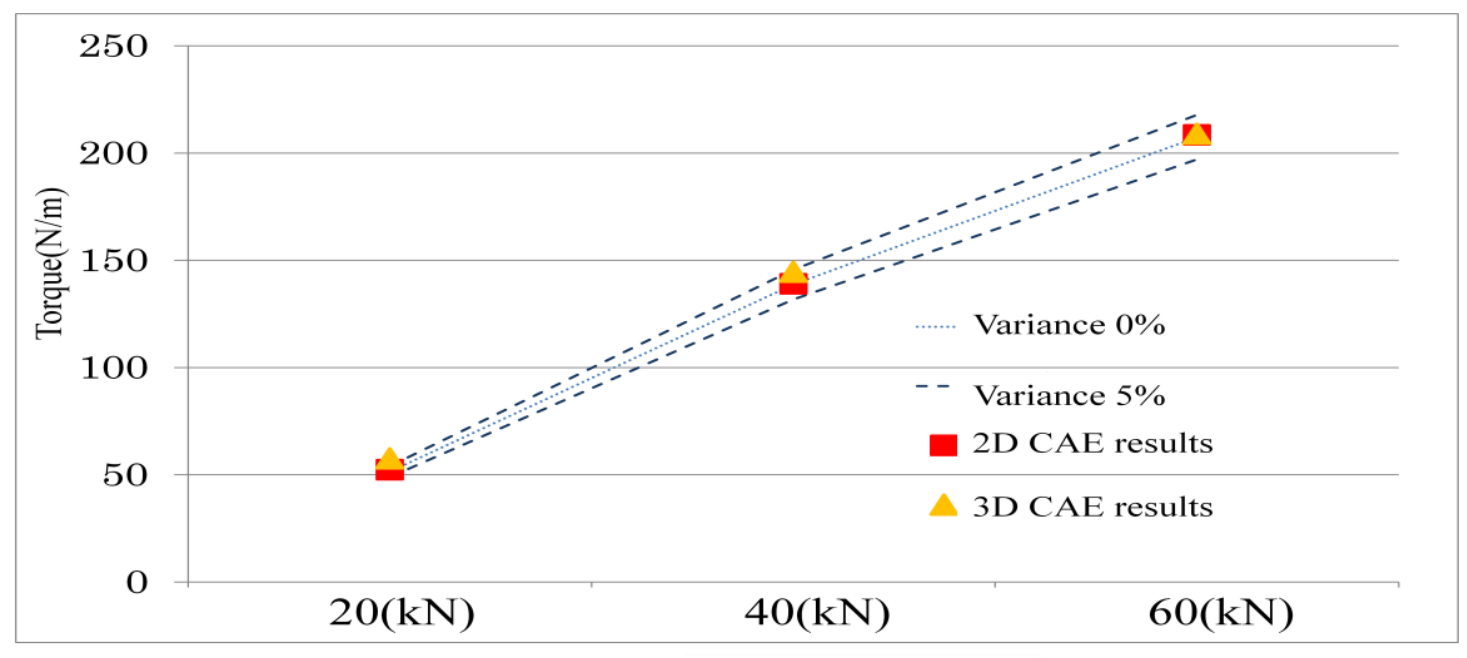

Axial force

Figure 13: Measuring the Friction Coefficient to Tighten Bolts 


\section{Comparison of Actual Product Testing and Three-dimensional CAE Analysis}

The results from actual product testing and three-dimensional CAE analysis shown in Figure 13 illustrate the relationship between torque and axial force. As can be seen in the diagram, the largest discrepancy between the two sets of results is 5\%. These results demonstrate the accuracy of CAE analysis and show that it is possible to reproduce almost the same results as those obtained from the testing of actual products.

\section{SUMMARY}

The authors believe that the use of statistical simulation technology in the form of CAE can contribute significantly to the regeneration of development and design processes, which is considered vital to the survival of Japan's automotive industry in the current situation. Thus, in this paper, the authors propose a changeover from the conventional prototyping and testing approach to a predictive evaluation process based on highly reliable CAE analysis. The highly reliable CAE analysis approach has been applied to bolt tightening analysis with significant results. This approach has been used to clarify issues related to the behavior of bolts during tightening through actual testing, and elements such as modeling, algorithms, reasoning, and calculation methods have been applied to establish the essential factors involved in bolt tightening analysis.

\section{AUTHOR INFORMATION}

Takashige Takahashi is a graduate student of the College of Science and Engineering at Aoyama Gakuin University.

Toshiya Ueno received his Master of Engineering degree from the College of Science and Engineering at Aoyama Gakuin University.

Manabu Yamaji is a research associate in the College of Science and Engineering at Aoyama Gakuin University, Japan. He received his Master of Engineering degree in Graduate School of Information Systems at University of Electro-Communications in 1999. His current research and teaching interests are in the general area of production engineering. In particular, he is interested in Total Quality Management, Computer Aided Engineering.

Kakuro Amasaka is a Professor in the College of Science and Engineering at Aoyama Gakuin University, Japan. $\mathrm{He}$ received his Ph.D. degree in Precision Mechanical and System Engineering, Statistics and Quality Control at Hiroshima University in 1997. His current research and teaching interests are in the general area of production engineering. In particular, he is interested in New JIT. He is a member of POMS and EurOMA.

\section{REFERENCES}

1. Amasaka, K, (2007a), Highly Reliable CAE Model, The Key to Strategic Development of New JIT, Journal of Advanced Manufacturing Systems, 6 (2): pp.159-176.

2. Amasaka, K., (2007b), High Linkage Model “Advanced TDS, TPS \& TMS”, Strategic Development of "New JIT" at Toyota -, International Journal of Operations and Quantitative Management, 13(3): pp. 101-121.

3. Amasaka, K, (2008), An Integrated Intelligence Development Design CAE Model Utilizing New JIT: Application to Automotive High Reliability Assurance, Journal of Advanced Manufacturing Systems, 7 (2): pp.221-241

4. Aragóna, A., Alegrea, J. and Gutiérrez-Solana F. (2005), Effect of Clamping Force on the Fatigue Behavior of Punched Plates Subjected to Axial Loading, Engineering Failure Analysis, 13(2): pp.271-281.

5. Baggerly, R. (1996). Hydrogen-Assisted Stress Cracking of High-Strength Wheel Bolts, Engineering Failure Analysis, 3(4):pp. 231-240.

6. Gamboa E. and Atrens A. (2003). Environmental Influence on the Stress Corrosion Cracking of Rock Bolts, Engineering Failure Analysis, 10(5): pp.521-558. 
7. Izumi, S., Yokoyama, T., Iwasaki, A. and Sakai, S. (2004). Three-Dimensional Finite Element Analysis of Tightening and Loosening Mechanism of Threaded Fastener, Engineering Failure Analysis, 12(4): pp.604-615.

8. Leea, H., Choia, J., Leea, B. and Kim, T. (2005). Failure Analysis of Stress Corrosion Cracking in Aircraft Bolts, Engineering Failure Analysis, 14(1):pp. 209-217.

9. Sakai, T. (2000). Introduction to conclusion of screw, Youkendou. (in Japanese)

10. Suzuki, M., ed. (2005). Encyclopedia of physics, Asakura-syoten. (in Japanese)

11. Tanaka, M., Miyazawa, H., Asaba, E. and Hongo, K. (1981). Application of the Finite Element Method to Bolt-nut Joints : Fundamental Studies on Analysis of Bolt-nut Joints Using the Finite Element Method, Bulletin of the Japan Society of Mechanical Engineers, 24(192): pp.1064-1071.

12. Ueno, T., Yamaji, M., Tsubaki, H., Amasaka, K., (2009), Establishment of Bolt Tightening Simulation System for Automotive Industry Application of the Highly Reliable CAE Model, International Business \& Economics Research Journal, 8 (5); pp.57-67.

13. Yamaji, M. and Amasaka, K., (2008), CAE Analysis Technology for Development Design Utilizing Statistical Sciences, The Open Industrial and Manufacturing Engineering Journal, 1; pp.1-8.

14. Yamaji, M. and Amasaka, K., (2009), Dual Technology Management Model of Design and Production utilizing CAE \& Experiment, The journal of China-USA Business Review, 8(4); pp.42-50. 
NOTES 